\title{
Effect of cobalt doping on the dielectric response of $\mathrm{Ba}_{0.95} \mathrm{~Pb}_{0.05} \mathrm{TiO}_{3}$ ceramics
}

\author{
E. Markiewicz • R. Bujakiewicz-Korońska • D. Majda • \\ L. Vasylechko • A. Kalvane $\cdot$ M. Matczak
}

Received: 15 January 2013 / Accepted: 9 September 2013 / Published online: 27 September 2013

(C) The Author(s) 2013. This article is published with open access at Springerlink.com

\begin{abstract}
Dielectric response of $\mathrm{Ba}_{0.95} \mathrm{~Pb}_{0.05} \mathrm{TiO}_{3}$ ceramics doped with 0.1 and 1 wt. $\%$ of $\mathrm{Co}_{2} \mathrm{O}_{3}$, synthesized by conventional high-temperature method, was studied in wide temperature and frequency range. The temperature dependences of the real and the imaginary parts of dielectric permittivity of the ceramics were compared with those of $\mathrm{BaTiO}_{3}$ and $\mathrm{Ba}_{0.95} \mathrm{~Pb}_{0.05} \mathrm{TiO}_{3}$. The addition of $\mathrm{Co}^{3+}$ ions results in a broadening of dielectric anomalies related to the transition to paraelectric cubic phase, and the structural transition between the tetragonal and the orthorhombic phases. At low temperatures (125-200 K) the dielectric absorption of Co-doped $\mathrm{Ba}_{0.95} \mathrm{~Pb}_{0.05} \mathrm{TiO}_{3}$ ceramics was found to exhibit relaxor-like properties. The dielectric response has been found to contain the contributions characteristic of fluctuations of the polar nanoregion boundaries and reorientations of the dipole moments between allowed directions in the nanoregions in the rhombohedral and the orthorhombic phases. The behavior speaks in favor
\end{abstract}

E. Markiewicz $(\bowtie) \cdot$ M. Matczak

Institute of Molecular Physics, Polish Academy of Science,

Smoluchowskiego 17, 60-179 Poznan, Poland

e-mail: ewamar@ifmpan.poznan.pl

R. Bujakiewicz-Korońska

Institute of Physics, Pedagogical University, Podchorążych 2,

30-084 Krakow, Poland

D. Majda

Department of Chemistry, Jagiellonian University, Ingardena 3,

30-060 Krakow, Poland

L. Vasylechko

Lviv Polytechnic National University, 12 Bandera, 79013 Lviv,

Ukraine

\section{A. Kalvane}

Institute of Solid State Physics, University of Latvia, Kengeraga 8, LV-1063 Riga, Latvia

M. Matczak

NanoBiomedicalCentre, Adam Mickiewicz University, Umultowska 85, 61-614 Poznan, Poland of ordering of polar defects in the host lattice of $\mathrm{Ba}_{0.95} \mathrm{~Pb}_{0.05} \mathrm{TiO}_{3}$ in a form polar nanoregions.

Keywords $\mathrm{Ba}_{0.95} \mathrm{~Pb}_{0.05} \mathrm{TiO}_{3} \cdot$ Co-doping $\cdot$ Dielectric properties $\cdot$ Deviation from the Curie-Weiss law

\section{Introduction}

$\mathrm{BaTiO}_{3}(\mathrm{BT})$ is a classic ferroelectric which has found wide application in the electronic industry. BT ceramics is commonly used in ceramic capacitors, piezoelectric sensors or actuators as well as resistive switching elements [1-3]. The compound can exist in four phases with cubic (above $398 \mathrm{~K}$ ), tetragonal (between 398 and $281 \mathrm{~K}$ ), orthorhombic (between 281 and $202 \mathrm{~K}$ ), and rhombohedral (below $202 \mathrm{~K}$ ) crystal structure $[4,5]$. All of the phases, except the cubic one, exhibit ferroelectric properties.

The structure and the dielectric properties of BT have been extensively studied for more than half a century in order to increase the dielectric permittivity and to suppress the losses $[6,7]$. Nowadays the research concentrated on temperaturestable dielectrics are intensely developed [8]. Pure BT is characterized by large variations in dielectric permittivity and dielectric losses related to the structural phase transitions. In order to avoid these disadvantages, doping of pure ceramics is used. BT is known to form solid solutions with $\mathrm{PbTiO}_{3}(\mathrm{PT})$ and an upward shift of the temperature of the tetragonal-cubic phase transition is observed with an increase of $\mathrm{Pb}$ content in $(1-x) \mathrm{BaTiO}_{3}-x \mathrm{PbTiO}_{3}$ solid solutions [9-13]. XAFS studies by Lebedev at al. [13] indicated a displacement of $\mathrm{Pb}$ atoms from the $A$ lattice site in the $A B \mathrm{O}_{3}$ perovskite structure by $\sim 0.15 \AA$ and a strong distortion of the oxygen environment as the main factors responsible for the appearance of ferroelectricity in the temperature range above $\sim 400 \mathrm{~K}$, that is the Curie point of pure $\mathrm{BaTiO}_{3}$ [14-17]. According to the 
literature data [4] the temperatures of the transitions from tetragonal to orthorombic and from orthorhombic to rhombohedral phases are lowered due to an addition of the $\mathrm{Pb}$ ions resulting in a broadening of the dielectric anomaly in the vicinity of room temperature.

Dielectric properties of the ceramics belonging to the $\mathrm{BaTiO}_{3}$ family can be influenced by doping with the transition-metal ions. It has been found that ions with the valences smaller than 4 substituting the Ti sites exhibit acceptor character with charge deficiency compensated by doubly ionized oxygen vacancies [1, 18]. Among the $3 \mathrm{~d}$ elements $\mathrm{Co}$ is known to influence the elastic and the dielectric properties of the $\mathrm{BaTiO}_{3}$ - based ceramics [2, $19,20]$. The addition of Co dopant up to 1 at.\% results in an attenuation of mechanical losses and a smoothing of the elastic modulus anomaly [2]. The decrease of mechanical losses in the orthorhombic phase is associated with the fixing of the domain wall positions by $\mathrm{Co}^{3+}$ acceptor ions. Dielectric measurement by H. J. Hagemann and H. Ihrig [1] showed the lowering of the Curie point with the content of Co dopants up to $2 \mathrm{~mol} \%$.

The aim of this paper is to characterize the effect of $\mathrm{Co}-$ doping on the dielectric response of $\mathrm{Ba}_{0.95} \mathrm{~Pb}_{0.05} \mathrm{TiO}_{3}$ solid solution with very low tetragonality (1-c/a) and to compare the response with those of the undoped ceramics and the $\mathrm{BaTiO}_{3}$ ceramics. We explore the impact of doping a material to modify the electrical properties, i.e. the relationship between the chemical and electrical properties. This relationship can be either in a good agreement with beneficial mechanical properties as pointed out in [2] or it can be coupled with lower piezoelectric activity [21]. The study of adjusting a given property can be easy adopted to the control of the another one. BTbased ceramics with $5 \%$ PT and $0.1 \%$ as well as $1 \% \mathrm{Co}_{2} \mathrm{O}_{3}$ doping can be very interesting from the application point of view because of the ability of a flattening the dielectric permittivity-temperature characteristic by the Co ions and a shift of the phase transition temperatures by the $\mathrm{Pb}$ dopants. $\mathrm{Co}^{3+}$ acceptor ions are incorporated into the $\mathrm{Ti}^{4+}$-lattice site because of their similar ionic radii and oxygen vacancies are generated in order to compensate the valence. The resultant acceptor-oxygen vacancy dipoles in highly polarizable lattice can form either polar nanoregions (PNRs) like in ferroelectric relaxors [22-24] or can result in an internal bias which is formally equivalent to the energy difference of the dipolar defects between the accessible directions of the spontaneous polarization and affects the dielectric response [25-27]. Due to high polarizability of the $\mathrm{ABO}_{3}$ lattice the dipolar defects polarize the neighboring part of the lattice forming the polar nanoregions the size of which is determined by temperature -dependent correlation length of the host. For low defect concentration each polar nanoregion can be treated as a noninteracting polar object characterized by a single relaxation time. At higher defect concentrations the PNRs can interact leading to more complex relaxational behavior apparent in so called ferroelectric relaxors as La substituted $\mathrm{PbZr}_{1-\mathrm{x}} \mathrm{Ti}_{\mathrm{x}} \mathrm{O}_{3}$ family or $\mathrm{PbMg}_{1 / 3} \mathrm{Nb}_{2 / 3} \mathrm{O}_{3}$ and $\mathrm{PbSc}_{1 / 2} \mathrm{Nb}_{1 / 2} \mathrm{O}_{3}$ families. The relaxor-like behavior is characterized by broad and highly dispersive dielectric anomaly [22-24].

Here we present the results of detailed studies of the dielectric response of $\mathrm{Co}_{2} \mathrm{O}_{3}$ doped $\mathrm{Ba}_{0.95} \mathrm{~Pb}_{0.05}$ ceramics to search for an influence of introduced defects on the equilibrium between the short-and the long range interactions in the host lattice. The material was characterized by X-ray diffraction (XRD) and differential scanning calorimetry (DSC).

\section{Experimental}

\subsection{Preparation of the samples}

BT - based ceramics with 5 at. $\%$ of $\mathrm{Pb}$ and 0.1 wt. $\%$ as well as 1 wt. $\%$ of $\mathrm{Co}_{2} \mathrm{O}_{3}$ were prepared by standard high temperature dry sintering method. Chemicals from Aldrich: $\mathrm{Co}_{2} \mathrm{O}_{3}$ (purity $99.5 \%), \mathrm{BaCO}_{3}(99.5 \%), \mathrm{PbO}(99.0 \%)$ and $\mathrm{TiO}_{2}(99.5 \%)$ were used. The $\mathrm{BaCO}_{3}$ and $\mathrm{TiO}_{2}$ powder were weighed in accordance to chemical formula stoichiometry to obtain $\mathrm{BaTiO}_{3}$ ceramics. The BPT and BPTC ceramics were also prepared in accordance to the presumed nominal compositions. The components were mixed in ethanol and homogenized in an agate ballmill for $24 \mathrm{~h}$. After drying at $400 \mathrm{~K}$, they were calcined for $2 \mathrm{~h}$ at the temperature of $1,493 \mathrm{~K}$. Then the calcined powders were grinded, pressed under the pressure of $15 \mathrm{MPa}$ at room temperature in form of pellets in diameter of $8 \mathrm{~mm}$, and sintered for $2 \mathrm{~h}$ at 1,593 K, at ambient air. The samples were cooled in the oven from sintering temperature to room temperature for $6 \mathrm{~h}$. EDX (Bruker - Quantax) studies of fractured BPTC samples show that the $\mathrm{Pb}$ amount lies in the range from $(5.38 \pm 0.22)$ to $(7.79 \pm 0.28)$ wt.\%. The samples in form of discs, thickness $d=1 \mathrm{~mm}$ and diameter $\phi=8 \mathrm{~mm}$, were cut off for dielectric measurements. The discs were covered with gold sputtered electrodes.

\subsection{XRD studies}

X-ray powder diffraction studies of the $\mathrm{Ba}_{0.95} \mathrm{~Pb}_{0.05} \mathrm{TiO}_{3}$ and $\mathrm{Ba}_{0.95} \mathrm{~Pb}_{0.05} \mathrm{TiO}_{3}+0.1 \% \mathrm{Co}_{2} \mathrm{O}_{3}$ ceramics were performed on the laboratory Huber imaging plate Guinier camera G670 with $\mathrm{Cu} K_{\alpha 1}$ radiation. The crystal structure of $\mathrm{Ba}_{0.95} \mathrm{~Pb}_{0.05} \mathrm{TiO}_{3}+$ $0.1 \% \mathrm{Co}_{2} \mathrm{O}_{3}$ and $\mathrm{Ba}_{0.95} \mathrm{~Pb}_{0.05} \mathrm{TiO}_{3}$ was determined from highresolution powder diffraction with synchrotron radiation in the temperature range $298-1,173 \mathrm{~K}$. Corresponding in situ hightemperature diffraction experiments were performed with powder diffractometer at the B2 beamline of synchrotron laboratory HASYLAB/DESY [28]. The program package WinCSD [29] was used in all crystallographic calculations.

\subsection{DSC measurements}

DSC measurements were carried out using a DSC $822^{\mathrm{e}}$ Mettler Toledo apparatus, equipped with liquid nitrogen cooling option 
Fig. 1 X-ray synchrotron powder diffraction patterns of $\mathrm{Ba}_{0.95} \mathrm{~Pb}_{0.05} \mathrm{TiO}_{3}$ (a) and $\mathrm{Ba}_{0.95} \mathrm{~Pb}_{0.05} \mathrm{TiO}_{3}: 0.1 \% \mathrm{Co}_{2} \mathrm{O}_{3}$ (b) $\lambda \lambda=0.53827 \AA$ at RT. Experimental (dots) and calculated patterns, difference profiles and positions of the diffraction maxima are given
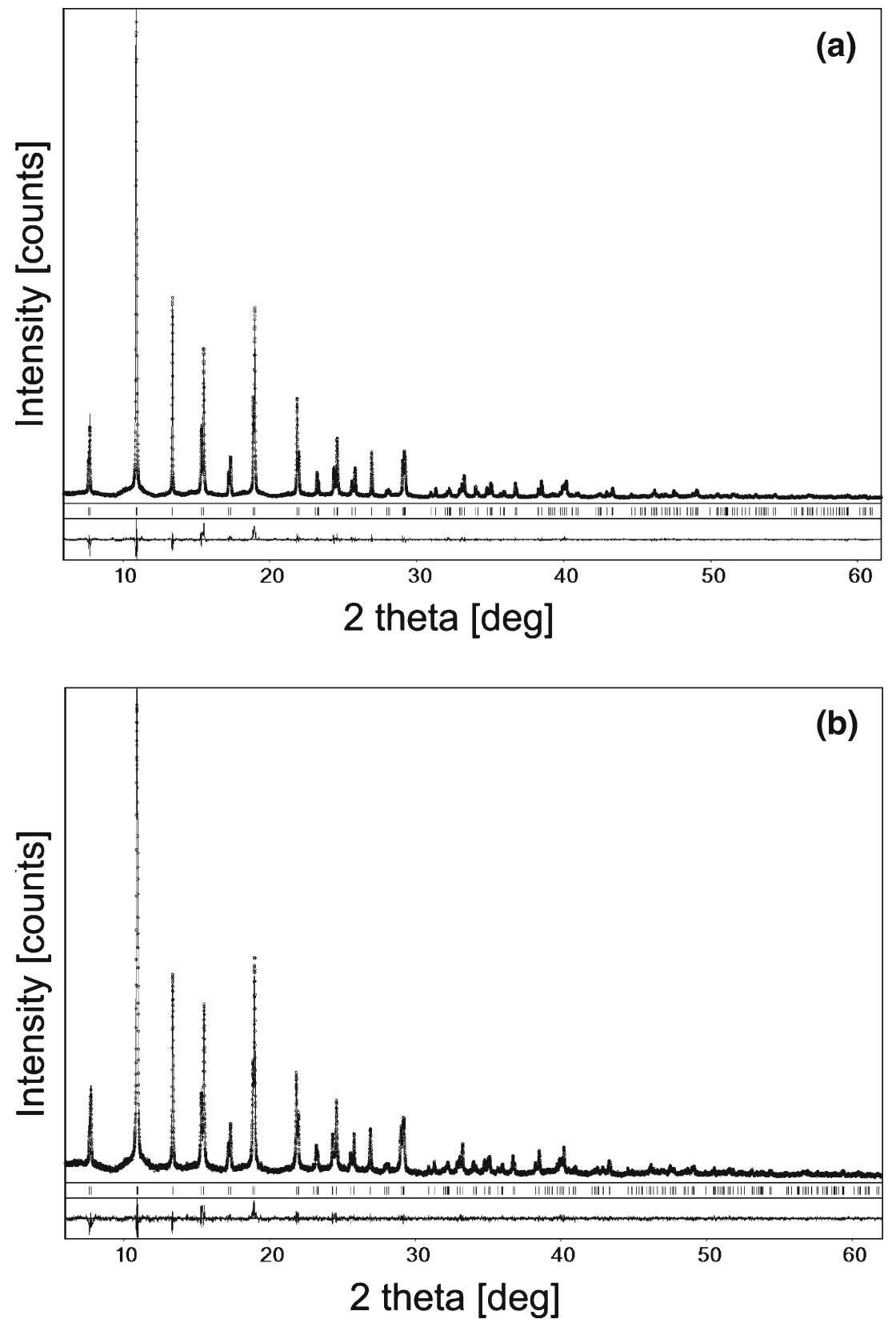

(Cryofab). The sample of studied material was hermetically sealed in an aluminum crucible and measured in temperature range from $123 \mathrm{~K}$ to $723 \mathrm{~K}$ at a heating rate of $10 \mathrm{~K} / \mathrm{min}$. The empty pan was used as a reference sample. The experiments were repeated twice to confirm the results.

\subsection{Dielectric measurements}

Dielectric response of the ceramic samples was studied using an Alpha-A High Performance Frequency Analyzer (Novocontrol $\mathrm{GmbH}$ ) combined with Quatro Cryosystem for the temperature control. The sample with gold sputtered electrodes was fixed between two additional external electrodes in a sample holder and placed into a cryostat. The measurements were performed in the temperature range from $125 \mathrm{~K}$ to $525 \mathrm{~K}$ on heating at a rate of $1 \mathrm{~K} / \mathrm{min}$. The frequency varied from $1 \mathrm{~Hz}$ to $1 \mathrm{MHz}$ at the oscillation voltage of $1 \mathrm{~V}$. The measured dielectric permittivity data were collected and evaluated by WinDETA impedance analysis software and a WinFit V 3.2. program.

\section{Results and discussion}

X-ray powder diffraction studies of the BPT and BPTC specimens at RT revealed tetragonal perovskite structure, similar to that of pure BT. Figure 1 shows the X-ray 
Table 1 Crystallographic data for $\mathrm{Ba}_{0.95} \mathrm{~Pb}_{0.05} \mathrm{TiO}_{3}: 0.1 \% \mathrm{Co}_{2} \mathrm{O}_{3}$ and $\mathrm{Ba}_{0.95} \mathrm{~Pb}_{0.05} \mathrm{TiO}_{3}$ at RT (space group $P 4 \mathrm{~mm}$ )
${ }^{\mathrm{a}} B_{\text {iso/eq }}=1 / 3\left[B_{11}(a *)^{2} a^{2}+\ldots\right.$ $\left.2 B_{23} b^{*} c * b c \cos a\right] ; \mathrm{T}=\exp [-1 /$ $4\left(B_{11}(a *)^{2} h^{2}+\ldots 2 B_{23} b * c *\right.$ $k l)$ ]

\begin{tabular}{ccccccccc}
\hline Atoms, sites & $x$ & $y$ & $z$ & $B_{\text {iso/eq }}{ }^{\mathrm{a}}, \AA^{2}$ & $B_{11}$ & $B_{22}$ & $B_{33}$ & $B_{12}, B_{13}, B_{23}$ \\
\hline $\mathrm{Ba}_{0.95} \mathrm{~Pb}_{0.05} \mathrm{TiO}_{3}: \mathrm{Co} ;$ & $a=3.99248(4) \AA, c=4.03373(6) \AA ;$ & $R_{\mathrm{I}}=0.064, R_{\mathrm{P}}=0.113$ \\
$\mathrm{Ba} / \mathrm{Pb}, 1 a$ & 0 & 0 & $0.0001(6)$ & $0.751(4)$ & $0.735(5)$ & $B_{11}$ & $0.783(9)$ & 0 \\
$\mathrm{Ti} / \mathrm{Co}, 1 b$ & $1 / 2$ & $1 / 2$ & $0.4884(7)$ & $0.75(1)$ & $0.77(1)$ & $B_{11}$ & $0.70(3)$ & 0 \\
$\mathrm{O} 1,1 b$ & $1 / 2$ & $1 / 2$ & $-0.019(2)$ & $0.83(6)$ & $0.90(10)$ & $B_{11}$ & $0.69(13)$ & 0 \\
$\mathrm{O} 2,2 c$ & $1 / 2$ & 0 & $0.512(2)$ & $0.85(5)$ & $0.94(10)$ & $0.74(7)$ & $0.89(8)$ & 0 \\
$\mathrm{Ba}_{0.95} \mathrm{~Pb}_{0.05} \mathrm{TiO}_{3} ; a=3.98898(9) \AA, c=4.0380(1) \AA ; R_{\mathrm{I}}=0.068, R_{\mathrm{P}}=0.131$ & & \\
$\mathrm{Ba} / \mathrm{Pb}, 1 a$ & 0 & 0 & $0.0001(9)$ & $0.756(8)$ & $0.71(1)$ & $B 11$ & $0.84(2)$ & 0 \\
$\mathrm{Ti}, 1 b$ & $1 / 2$ & $1 / 2$ & $0.4848(9)$ & $0.68(4)$ & $0.73(2)$ & $B 11$ & $0.57(12)$ & 0 \\
$\mathrm{O} 1,1 b$ & $1 / 2$ & $1 / 2$ & $-0.014(4)$ & $0.75(14)$ & $0.83(13)$ & $B 11$ & $0.6(4)$ & 0 \\
$\mathrm{O} 2,2 c$ & $1 / 2$ & 0 & $0.518(2)$ & $0.8(2)$ & $1.4(2)$ & $0.4(1)$ & $0.7(4)$ & 0 \\
\hline
\end{tabular}

synchrotron powder diffraction patterns and the results of full profile Rietveld refinement obtained for both specimens. The final values of structural parameters are presented in Table 1. Besides the lattice parameters, the atomic coordinates and anisotropic displacement parameters of atoms were refined.

Examination of diffraction patterns of BPTC and BPT collected at the elevated temperatures shows that tetragonal deformation decreases with increasing temperature and completely vanishes at the temperatures higher than $453 \mathrm{~K}$ and $473 \mathrm{~K}$ for BPTC and BPT, respectively (Fig. 2). The patterns collected above this temperature show pure cubic perovskite structure. Accordingly, crystal structure parameters of the tetragonal and cubic phases of both samples have been refined in space groups $P 4 m m$ and $P m \overline{3} m$, respectively. Detailed examination of the diffraction patterns of BPTC collected in the vicinities of the transition temperature revealed a coexistence of both tetragonal and cubic phases in the temperature range $383 \mathrm{~K}-423 \mathrm{~K}$ (inset in Fig. 2), which proves the 1st-order discontinuous character of the phase transition in BPTC. Similar behaviour was also observed for the BPT crystal.

Simultaneous two-phase Rietveld refinement performed in space groups $P 4 \mathrm{~mm}$ and $P m \overline{3} m$ confirms a coexistence of tetragonal and cubic perovskite phases in the vicinities of the transition temperatures. According to the data obtained, the amount of the tetragonal phase in BPTC decreases from 90.0 wt. $\%$ at $383 \mathrm{~K}$ to $20.5 \mathrm{wt} . \%$ at $423 \mathrm{~K}$, whereas the content of the cubic phase simultaneously increases.

Figure 3 demonstrates the change in relative amounts of both perovskite phases in BPTC and BPT at the phase transitions. From these plots the temperatures of the phase transitions (the temperatures at which the amounts of tetragonal and cubic phases are equal) can be estimated as $410 \mathrm{~K}$ and $442 \mathrm{~K}$, respectively.

Temperature dependence of the lattice parameters and cell volumes of tetragonal and cubic modifications of BPTC and BPT illustrating the first-order structural phase transition are presented in Fig. 4. The microstructural parameters of BPT
Fig. 2 Fragments of diffraction patterns collected in situ in the temperature range 343-473 K with the temperature step of $10 \mathrm{~K}$ (from the bottom to the top) illustrating the structural phase transition in BPTC $(0.1 \%)$

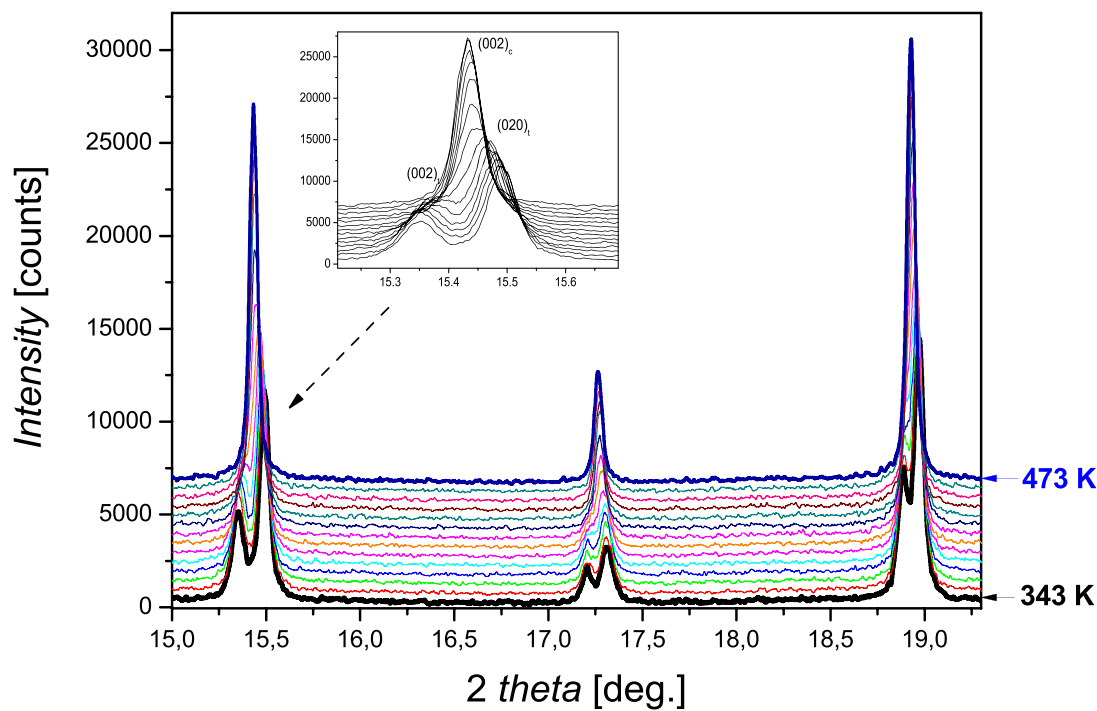



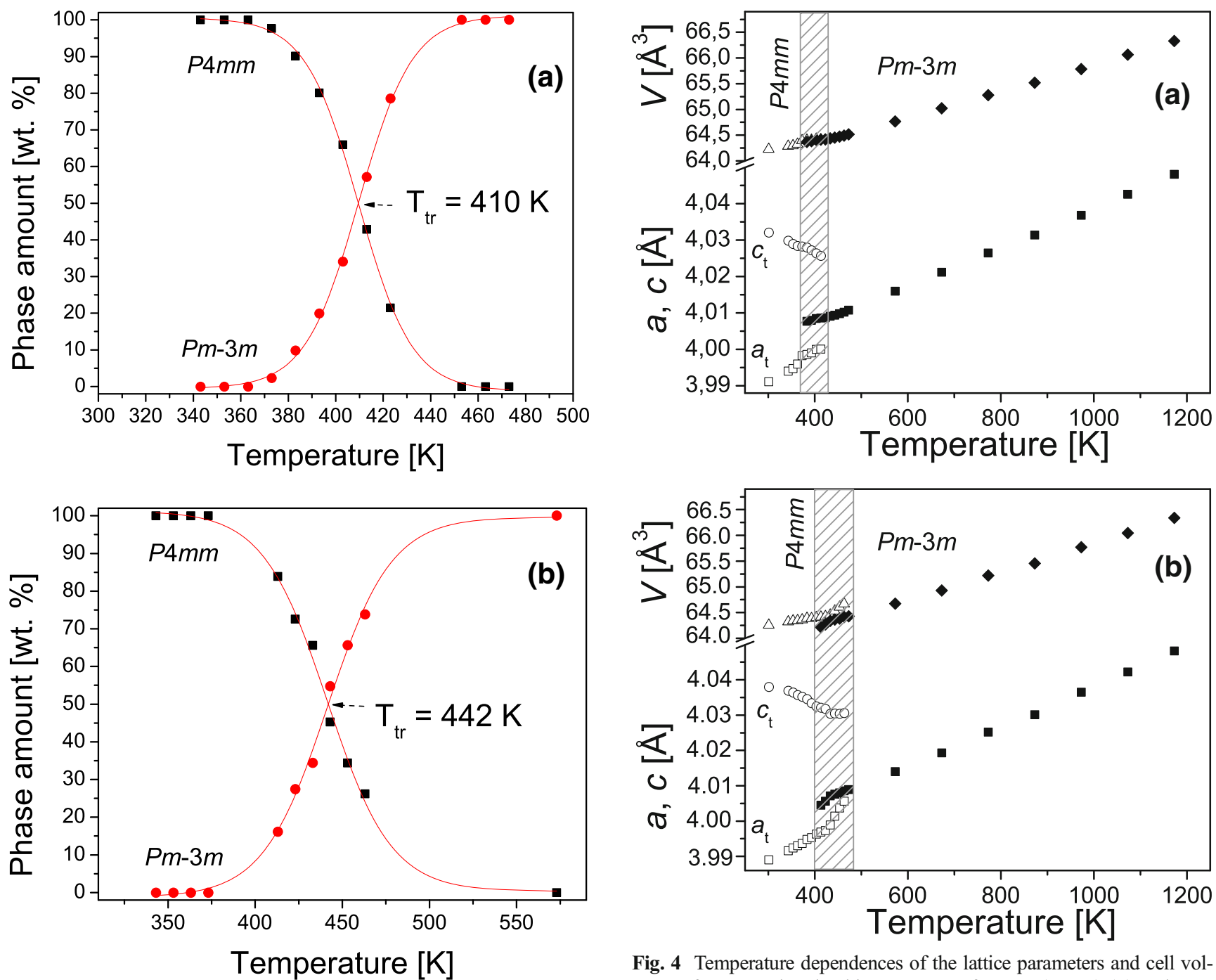

Fig. 3 Refined values of the amount of tetragonal and cubic perovskite phases in BPTC $(0.1 \%)(\mathbf{a})$ and BPT (b) in the temperature range of the phase transitions

and BPTC samples (average grain size $\mathrm{D}_{\text {ave }}$ and microstrains parameters $\langle\varepsilon\rangle=\langle\Delta d>/$ d) were obtained from the analysis of the broadening of X-ray powder diffraction maxima by Williamson-Hall analysis, which allows to separate the effect of size and strain broadening due to their different dependence on the scattering angle. For the BPT and BPTC $(0.1 \%)$ samples the X-ray synchrotron powder diffraction data were collected at beamline B2 synchrotron laboratory HASYLAB/ DESY and the $\mathrm{LaB}_{6}$ external standard was used for determination of instrumental broadening of the diffraction maxima. Graphical results of Williamson-Hall analysis of the microstructure of BPT and BPTC samples are presented in Fig. 5. The analysis of angular dependence of the line with (HWFM) shows that the samples are characterized by similar microstructure parameters. The average grain size was assessed to be of several hundred $\mathrm{nm}$, and the average microstrains parameters $<\varepsilon>$ are between 0.04 and $0.05 \%$.

Fig. 4 Temperature dependences of the lattice parameters and cell volumes of tetragonal and cubic structures of BPTC (0.1\%) (a) and BPT (b). The areas of coexistence of both perovskite phases are dashed

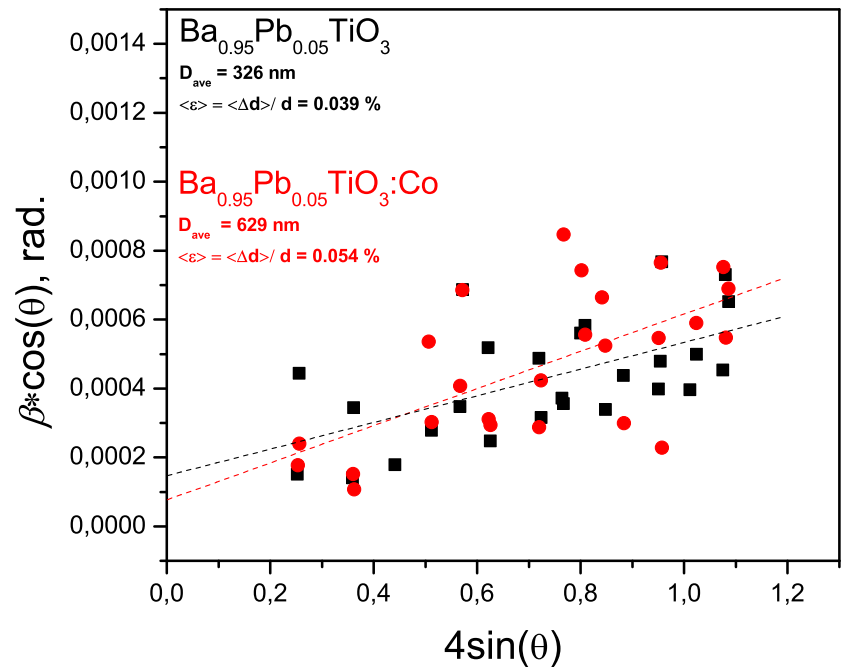

Fig. 5 Graphical results of the Williamson-Hall analysis of microstructure of the BPT and BPTC $(0.1 \%)$ samples by using synchrotron X-ray powder diffraction data (beamline B2 at HASYLAB/DESY, $\lambda=$ $0.53821 \AA, \mathrm{LaB}_{6}$ standard, $\mathrm{HWFM}_{\mathrm{St}}=0.058-0.065 \mathrm{deg}$ ) 


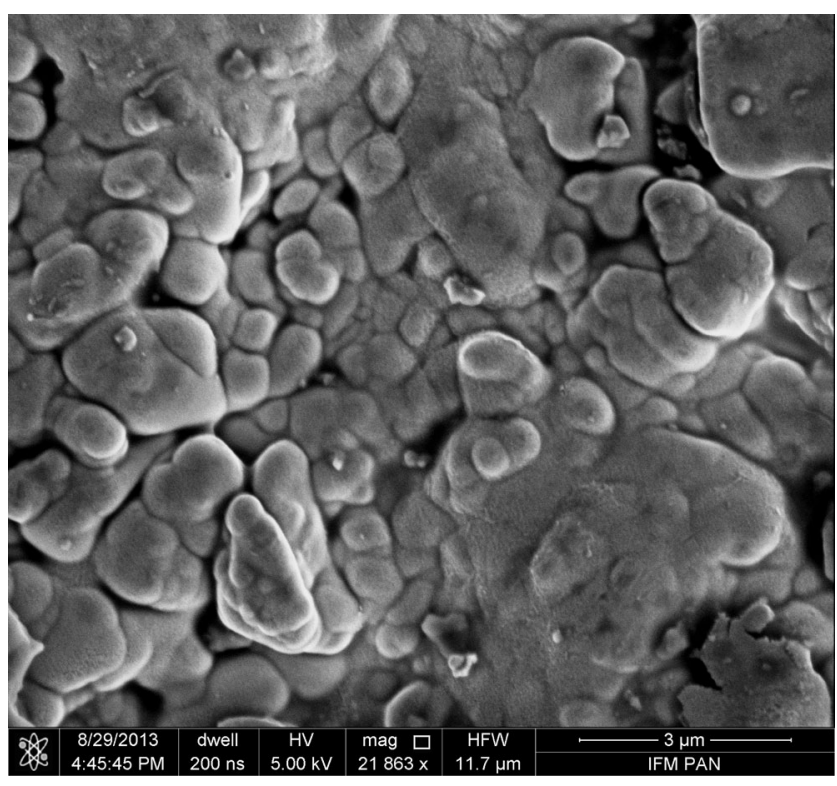

Fig. 6 SEM image of the fracture of $\operatorname{BPTC}(0.1 \%)$ ceramic sample

Figure 6 shows an example of the scanning electron microscope (SEM) image of a fracture of BPTC $(0.1 \%)$ sample. One can observe individual grains of average sizes of several hundred $\mathrm{nm}$ and their agglomerates.

The results of DSC measurements (Fig. 7) sustain the 1storder discontinuous character of the Curie phase transition in BPT. At $430 \mathrm{~K}$, one can observe a sharp peak, shifted by about $30 \mathrm{~K}$ towards higher temperatures in comparison with that of the BT sample. Both structural transitions: between the tetragonal and the orthorhombic phases as well as from the orthorhombic phase to the rhombohedral one in BPT are lowered by $\sim 40 \mathrm{~K}$. In the case of BPTC, all phase transitions were found to be diffused.

The enthalpy $\Delta H$ related to particular transitions in $\mathrm{BT}$ and BPT can be evaluated from the excess specific heat capacity using the following equation [30]:

$\Delta H(T)=\int_{T_{1}}^{T_{2}} C_{p}(T) d T$.

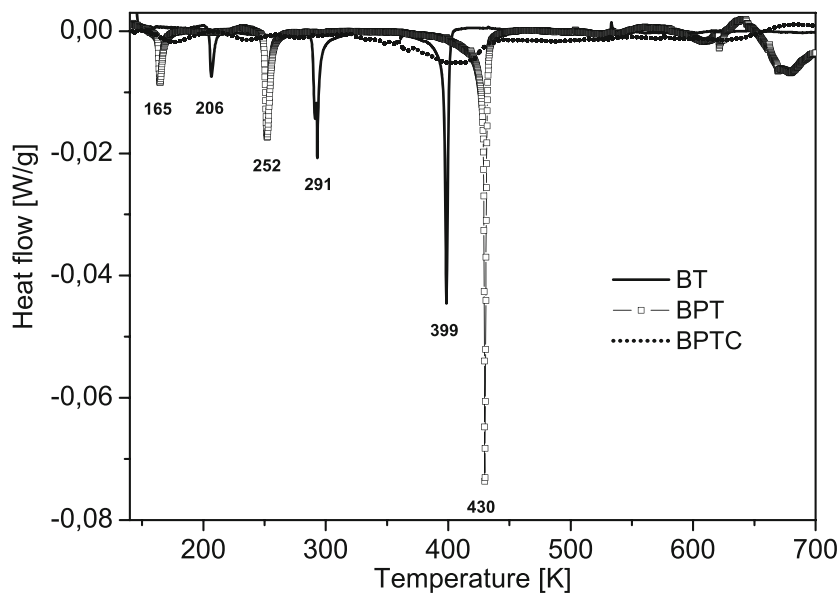

Fig. 7 DSC curves of BT, BPT and BPTC $(0.1 \%)$ ceramics
Table 2 The enthalpy $\Delta H$ obtained for BT and BPT

\begin{tabular}{lll}
\hline Substance & $\begin{array}{l}\text { Temperature of occurrence } \\
\text { of peaks } T_{c} ; T_{1} ; T_{2}[\mathrm{~K}]\end{array}$ & $\Delta H[\mathrm{~J} / \mathrm{mol}]$ \\
\hline BT & 399 & 275 \\
& 291 & 163 \\
& 206 & 37 \\
BPT & 430 & 426 \\
& 252 & 130 \\
& 165 & 50 \\
\hline
\end{tabular}

The values of $\Delta H$ calculated for BT and BPT are summarized in Table 2.

Figure 8 shows temperature variation of the real part of the permittivity measured at the frequency of $1 \mathrm{kHz}$ for BT, BPT and BPTC ceramics. One can observe a shift of $T_{c}$ towards higher temperatures by about $30 \mathrm{~K}$ in the case of BPT in comparison with that of BT. The result coincides with those of our DSC studies. The addition of $\mathrm{Co}^{3+}$ ions results in a broadening of the dielectric anomaly and a downward shift of the Curie point. In the case of $0.1 \%$ doping with $\mathrm{Co}_{2} \mathrm{O}_{3}$ the Curie temperature appears to be shifted by $\sim 5.5 \mathrm{~K}$ and the dielectric anomaly is considerably lower than that of BPT ceramics. BPTC doped with $1 \%$ of $\mathrm{Co}_{2} \mathrm{O}_{3}$ shows a huge dielectric anomaly shifted downwards by $\sim 34 \mathrm{~K}$ with respect to that of BPT. The effect confirms the observation by H. J Hagemann and $\mathrm{H}$. Ihrig [1] for $\mathrm{BaTiO}_{3}$ polycrystal doped with $\mathrm{Co}^{3+}$.

All investigated materials do not obey the Curie-Weiss law above $T_{C}$ (Fig. 9). The temperature range $\Delta T_{c m}$ in which the deviation appears was estimated as the difference in the temperature at which the experimental point diverges from the CurieWeiss law and the temperature of minimum of $1 / \varepsilon^{\prime}$. The accuracy of the $\Delta T_{c m}$ estimation we assess as $\pm 0.2 \mathrm{~K}$. This behavior points to the mechanism of the paraelectric-ferroelectric transition composed of two contributions: the displacive and the order-

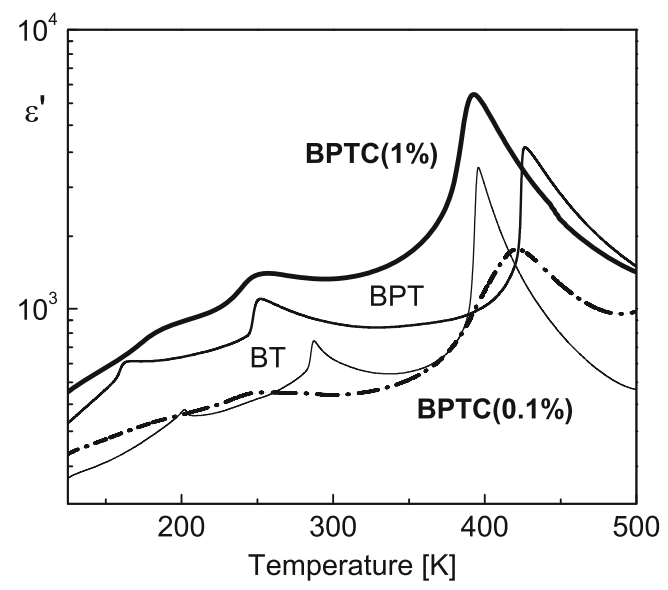

Fig. 8 Temperature dependences of real part of dielectric permittivity $\varepsilon^{\prime}$ obtained for BT, BPT, $\operatorname{BPTC}(0.1 \%)$ and $\mathrm{BPTC}(1 \%)$ ceramics at the frequency of $1 \mathrm{kHz}$ 


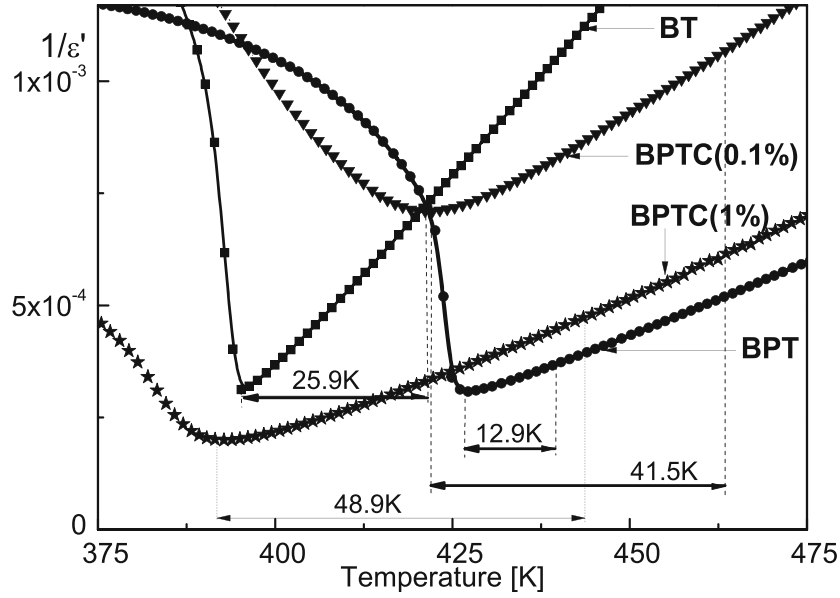

Fig. 9 Deviation degree $\Delta T c m$ from the Curie-Weiss law at the frequency of $100 \mathrm{kHz}$ obtained for BT, BPT, BPTC(0.1\%) and BPTC(1\%) ceramics

disorder one. In the barium titanate the central Ti ions jump between eight equivalent $<111>$ off-center sites inside the oxygen octahedra [31]. Fast hopping of Ti ions coexists with slow $90^{\circ}$ flipping of displacive soft mode induced tetragonal nanodomains in the paraelectric phase [32]. As a consequence, polar clusters appear and grow when approaching the phase transition from the high temperature side. A lot of experimental methods was used to evidence both displacive and orderdisorder character of the mechanism of conducting the Curie phase transition. The measurements of temperature dependence of the birefringence revealed the $\Delta n$ magnitude of the order of $10^{-5}$ up to about $30 \mathrm{~K}$ above $T c$ due to the presence of polar clusters $[33,34]$. NMR observation of the first order satellites in ${ }^{47} \mathrm{Ti}$ and ${ }^{49} \mathrm{Ti}$ spectra in the cubic phase of $\mathrm{BaTiO}_{3}$ showed the

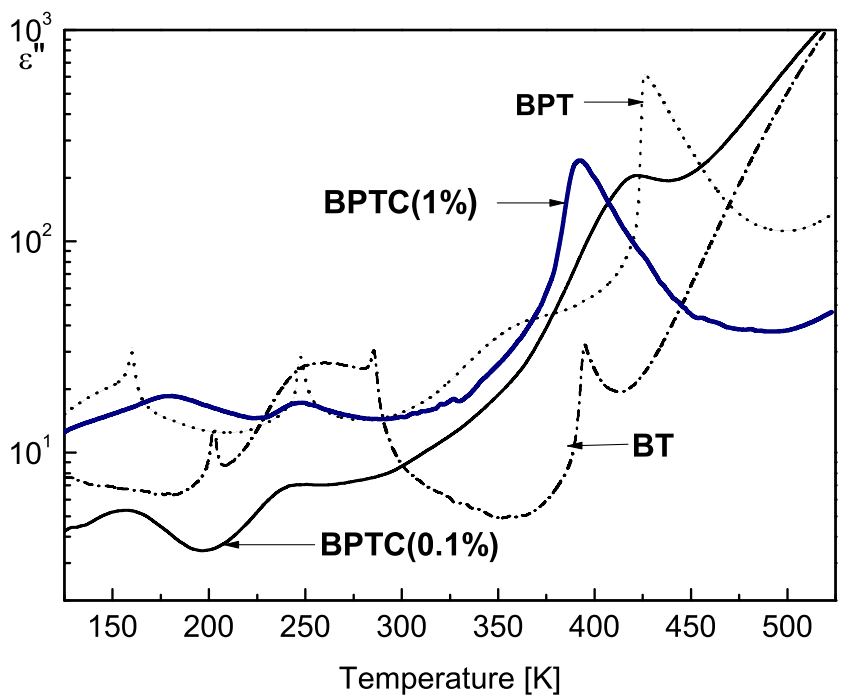

Fig. 10 Temperature dependences of imaginary part of dielectric permittivity $\varepsilon^{\prime \prime}$ obtained for BT, BPT, BPTC $(0.1 \%)$ and BPTC $(1 \%)$ ceramics at the frequency of $1 \mathrm{kHz}$ presence of nonzero quadrupole coupling at the Ti sites $[35,36]$ pointing to a combined displacive and order-disorder mechanism of the Curie transition. The existence of polar clusters in the paraelectric phase influences also the electrostrictive properties of $\mathrm{BaTiO}_{3}$ because of the nonzero value of squared polarization $P^{2}$
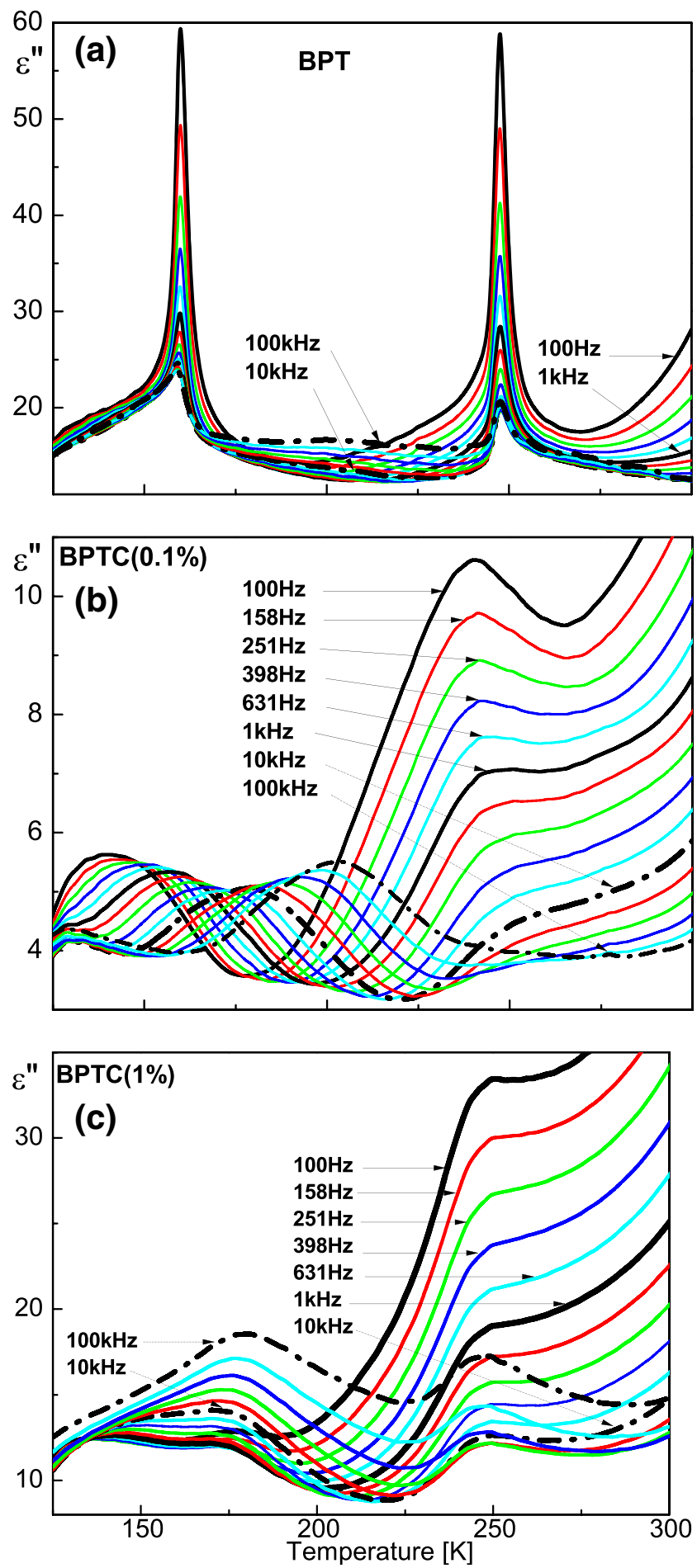

Fig. 11 Dielectric absorption of BPT, BPTC $(0.1 \%)$ and $\operatorname{BPTC}(1 \%)$ ceramics in the low temperature range 


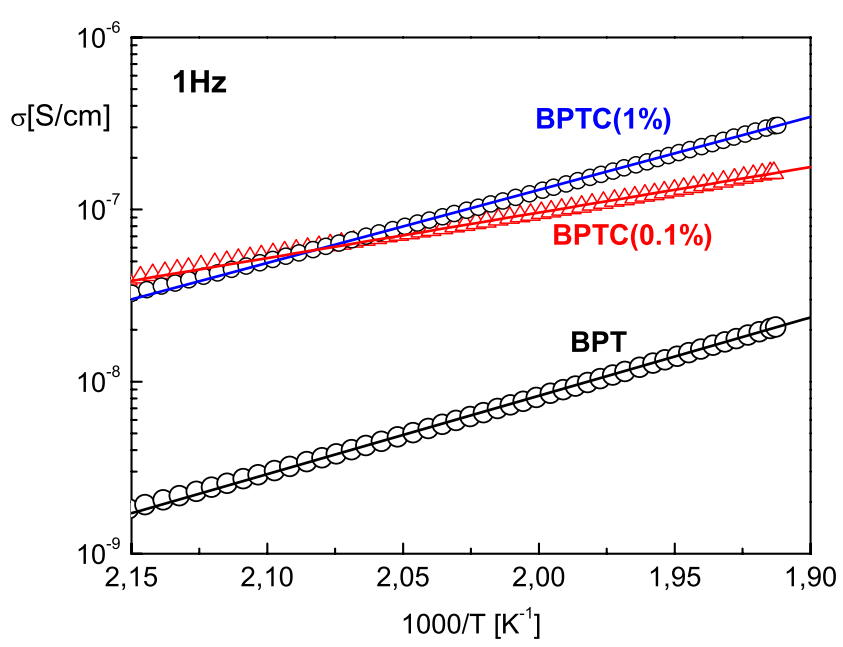

Fig. 12 Arrhenius plots of electric conductivity of BPT, BPTC $(0.1 \%)$ and $\mathrm{BPTC}(1 \%)$ ceramics measured at the frequency of $1 \mathrm{~Hz}$ in the temperature range $465 \mathrm{~K}-525 \mathrm{~K}$

despite the fact that the remnant polarization is not measurable [37]. Brillouin light scattering studies [38, 39] showed anomalous changes of acoustic phonons accompanied by the appearance of quasielastic central peak on approaching $T c$ in the paraelectric phase. Moreover, many theoretical works based on the first-principle calculations and polarizability model [40-42] have been done to explain the deviation from Curie-Weiss behavior due to the complex dynamics of the transition.

The dielectric permittivity-temperature characteristic of BPTC shows the highest deviation degree from the CurieWeiss law measured as $\Delta T_{c m}=T_{d e v}-T_{m}$ [43], where $T_{d e v}$ denotes the temperature above which $1 / \varepsilon^{\prime}$ starts to obey the Curie-Weiss law. However the lowest deviation can be noticed for BPT. Probably, the origin of this effect is higher tetragonality $(c / a=1.012)$ observed for PBT by the XRD study (Table 1$)$ in comparison with that of BT $(c / a=1.011)[44,45]$ and BPTC $(1 \%)$ $(c / a=1.010)$. The deviations $\Delta T_{c m}$ determined on the basis of the $1 / \varepsilon^{\prime}$ dependence on temperature at the frequency of $100 \mathrm{kHz}$ shown in Fig. 9 are: $25.9 \mathrm{~K}, 12.9 \mathrm{~K}, 41.5 \mathrm{~K}$ and $48.9 \mathrm{~K}$ for BT, BPT, BPTC (0.1\%) and BPTC(1\%), respectively.

As seen in Fig. 8 the dielectric anomalies associated with structural transitions: from the tetragonal to the orthorhombic and from the orthorhombic to the rhombohedral phases are also influenced by doping of BT with $\mathrm{Pb}^{2+}$ and $\mathrm{Co}^{3+}$ cations. For the BPT one can observe a shift of the anomalies by about $40 \mathrm{~K}$ towards lower temperatures. However, the doping of BPT with $\mathrm{Co}^{3+}$ cations results in diffuseness of the anomaly related to the transition between the tetragonal and the orthorhombic phases. The transition from the orthorhombic phase to the rhombohedral one was not observed in the investigated temperature and frequency ranges.

Temperature dependences of the imaginary parts of dielectric permittivity $\varepsilon^{\prime \prime}$ shown in Fig. 10 enable further explanation of the effect of dopants on the dielectric properties of BPTC. For BPT one can observe the maxima of dielectric permittivity $\varepsilon^{\prime \prime}$ bellow the Curie transition. The maxima are related to the contribution of domain wall motion to the dielectric response. The characteristic features of this process are the following: 1) it can appear only in the ferroelectric phase; 2) the relaxation frequency is strongly temperature dependent only very close to $T_{C}$ and far below it approaches a value which is sample dependent; 3 ) the strength is strongly dependent on the quality of the material so in the case of high defect concentration it can vanish entirely [46]. In the ceramics studied, the defects can be located on the domain walls and pin their motion in the external electric field [47]. After doping with $\mathrm{Co}^{3+}$ cations, i.e. for BPTC one should notice the reduction of dielectric losses attributed to the domain wall motion in the orthorhombic phase. These results are in a good agreement with that obtained from the mechanical results published in [2]. We can confirm the remarks by Hardtl [48] that acceptor dopants ions fix the domain wall position.

The situation appears to be more complicated for $\mathrm{Co}^{3+}$ doped PBT ceramics in the low-temperature region as shown in Fig. 11. The phase transition from the rhombohedral to the orthorhombic structure apparent as $\varepsilon^{\prime \prime}(f, T)$ anomaly at $\sim 160 \mathrm{~K}$ for $\mathrm{Ba}_{0.95} \mathrm{~Pb}_{0.05} \mathrm{TiO}_{3}$ disappears for cobalt doped ceramic and in the same temperature range relaxor-like relaxation processes are observed. In the case of BPTC $(0.1 \%)$ samples the maximum values of the imaginary part of dielectric permittivity $\varepsilon^{\prime \prime}{ }_{\text {max }}$ decrease with increasing frequency in the window $100 \mathrm{~Hz} \leq f \leq 6 \mathrm{kHz}$ whereas at higher frequencies an increase in $\varepsilon^{\prime \prime}{ }_{\text {max }}$ is observed and at $100 \mathrm{kHz}$ its maximum appears at $T_{\varepsilon^{\prime \prime} \text { max }} \approx 203 \mathrm{~K}$. According to the theory of the dielectric response of ferroelectric relaxors [49, 50], which considers the behavior of $\varepsilon^{\prime}{ }_{\text {max }}$ and $\varepsilon^{\prime \prime}{ }_{\text {max }}$ with increasing frequency, i) in the case when $\varepsilon^{\prime}{ }_{\text {max }}$ decreases and $\varepsilon^{\prime \prime}$ max $_{\text {max }}$ increases with increasing frequency the main contribution to the dielectric response is due to flipping of the dipole moments between allowed directions in the polar nanoregions (PNRs), ii) whereas a decrease in $\varepsilon_{\text {max }}^{\prime}$ and $\varepsilon^{\prime \prime}{ }_{\text {max }}$ with increasing frequency is related to the contribution of fluctuations of the boundaries of PNRs. Thus we would like to relate the dielectric response observed in the frequency range $100 \mathrm{~Hz}-6 \mathrm{kHz}$ to be due mainly to the contribution of fluctuations of PNR boundaries, whereas that apparent in the frequency range $10 \mathrm{kHz}-100 \mathrm{kHz}$ to the response of the dipole moments in the dynamic polar nanoregions. The contribution of the fluctuations of the boundaries of PNRs in BPTC $(1 \%)$ is visible in the frequency window $100 \mathrm{~Hz}-1 \mathrm{kHz}$ only and above $1 \mathrm{kHz}$ the contribution of dipole moments flipping between allowed $<111>$ cub directions in PNRs dominates in $\varepsilon^{\prime \prime}(f, T)$ behavior with $T_{\varepsilon^{\prime \prime} \text { max }} \approx 180 \mathrm{~K}$ at $100 \mathrm{kHz}$.

The transition from the phase with the orthorhombic structure to that with the tetragonal symmetry appears as a narrow $\varepsilon^{\prime \prime}$ peak at $247.5 \mathrm{~K}$ in pure $\mathrm{Ba}_{0.95} \mathrm{~Pb}_{0.05} \mathrm{TiO}_{3}$ ceramics which in $\mathrm{Co}^{3+}$ doped ceramics becomes considerably broadened. In BPTC (1\%) samples at frequencies higher than $\sim 10 \mathrm{kHz}$ an additional contribution to the dielectric absorption, most 
probably due to the dipole moments flipping between allowed $\langle 110\rangle_{\text {cub }}$ directions, can be distinguished. We did not determine the activation energies $E_{a}$ of the processes because the measurements were done in a narrow frequency window (2-3 frequency decades) and the results of fitting to Vogel-Fulcher equation would be loaded with a great error.

To characterize the effect of $\mathrm{Co}^{3+}$ doping on the high temperature electric properties of BPTC ceramics we show in Fig. 12 Arrhenius plots of electric conductivity of the ceramic samples measured at the frequency of $1 \mathrm{~Hz}$ in the temperature range $465 \mathrm{~K}-525 \mathrm{~K}$. Doping results in an increase in the conductivity by more than one order of magnitude due to oxygen vacancies introduced to compensate the $\mathrm{Co}^{3+}$ valence at the B-site of the perovskite lattice. The activation energies of the oxygen vacancies migration were assessed to amount to $(0.90 \pm 0.05) \mathrm{eV},(0.84 \pm 0.05) \mathrm{eV}$ and $(0.052 \pm 0.05) \mathrm{eV}$, respectively for BPT, BPTC $(1 \%)$ and BPTC $(0.1 \%)$ ceramics. The activation energy of free oxygen vacancy migration in ferroelectric perovskite oxides has been reported to be of $0.8-1 \mathrm{eV}$ $[51,52]$ thus we can relate the high-temperature electric conduction determined for BPT and BPTC $(1 \%)$ ceramics to the process of oxygen vacancies migration. As the distribution of the oxygen vacancies in the lattice has been considered in the tetragonal phase of the ferroelectric perovskites only $[26,50$, 52 ] it is hard to discuss the lowering of the activation energy for $\operatorname{BPTC}(0.1 \%)$ since the energy landscape of oxygen and probably also $\mathrm{Pb}$ vacancies is uncertain.

\section{Conclusions}

A new ceramic material (BPTC) was proposed for applications in the electronic industry and its dielectric properties were investigated. The experiment confirmed that the content of $\mathrm{Pb}^{2+}$ ions substituting the $\mathrm{Ba}^{2+}$ ions in $A$ - site is very useful tool to control the Curie temperature. From the application point of view it is very promising the effect of reduction of high dielectric losses related to the structural phase transition in BPTC due to doping with $\mathrm{Co}^{3+}$ ions. It should be mentioned that $\mathrm{Pb}^{2+}$ and $\mathrm{Co}^{3+}$ doping of $\mathrm{BaTiO}_{3}$ results in a diffusiveness and broadening of the ferroelectric - paraelectric phase transition. Moreover, we found that BPTC exhibits relaxor-like behavior in the low-temperature range, from 125 to $225 \mathrm{~K}$ which speaks in favour of the polar nanoregions, originating from the presence of $\mathrm{Co}^{3+}$ acceptor-oxygen vacancy dipoles in highly polarizable lattice, to be responsible for the modification of the physical properties. Moreover, the dielectric relaxation processes apparent in the rhombohedral and the orthorhombic phases of Co-doped BPT ceramics enabled to distinguish the contribution to the dielectric response of fluctuations of polar nanoregion boundaries and that of dipole moments reorientation between the allowed directions in the polar nanoregions. As the Codoping has been reported to decrease the mechanical losses and to smooth the elastic modulus anomaly in BT based ceramics [2] we believe to open the discussion on the effect of a well defined polar heterogeneity at the nanoscale in oxide perovskites on the anomalies of physical properties at the structural phase transitions.

Acknowledgments The dielectric response studies were supported by the funds from COST Action MP0904: Single- and multiphase ferroics and multiferroics with restricted geometries (SIMUFER). The part of the DSC measurements was carried out with the equipment purchased thanks to the financial support of the European Regional Development Fund in the framework of the Polish Innovation Economy Operational Program (contract no. POIG.02.01.00-12-023/08). L. Vasylechko acknowledges a support of the Ministry of Education, Science, Youth and Sports of Ukraine (projects DB/Neos) and the ICDD Grant-in-Aid program. In situ high - temperature diffraction measurements were carried out during beamtimes allocated to HASYLAB Project I-20110214.

Open Access This article is distributed under the terms of the Creative Commons Attribution License which permits any use, distribution, and reproduction in any medium, provided the original author(s) and the source are credited.

\section{References}

1. H.J. Hagemann, H. Ihring, Valence change and phase stability of $3 d-$ doped $\mathrm{BaTiO}_{3}$ annealed in oxygen and hydrogen. Phys. Rev. B 20 , 3871-3878 (1979)

2. B.L. Cheng, M. Gabbay, W. Jr Duffy, G. Fantozzi, Mechanical loss and Young's modulus associated with phase transitions in barium titanate based ceramics. J. Mater. Sci. 31, 4951-4955 (1996)

3. K. Uchino, Ferroelectric Devices (Marcell Dekker, Inc., New York Basel, 2000), pp. 105-118

4. Landolt-Börnstein, Numerical Data and Functional Relationships in Science and Technology, Vol. III/16a (Springer, Berlin, 1981), p. 421

5. Y.L. Li, L.E. Cross, L.Q. Chen, A phenomenological thermodynamic potential for $\mathrm{BaTiO}_{3}$ single crystals. J. Appl. Phys. 98, 064101-1-4 (2005)

6. R.F. Blunt, W.F. Love, The dielectric properties of Barium Titanate at low temperatures. Phys. Rev. 76, 1202-1204 (1949)

7. G. Arlt, D. Hennings, G. de With, Dielectric properties of fine-grained barium titanate ceramics. J. Appl. Phys. 58, 1619-1625 (1985)

8. H. Kishi, Y. Okino, M. Honda, Y. Iguchi, M. Imaeda, Y. Takahashi, H. Ohsato, T. Okuda, The effect of $\mathrm{MgO}$ and rare-earth oxide on formation behavior of core-shell structure in $\mathrm{BaTiO}_{3}$. Jpn. J. Appl. Phys. 36, 5954-5957 (1997)

9. J.F. Meng, B.K. Rai, R.S. Katiyar, G.T. Zou, Study of visible emission and phase transition in nanocrystalline $\mathrm{A}_{(1-\mathrm{x})} \mathrm{A}_{\mathrm{x}}^{\prime} \mathrm{TiO}_{3}$ systems. Phys. Lett. A 229, 254-258 (1997)

10. A.V. Myasoedov, S.R. Syrtsov, Positive temperature coefficient of resistance in the lead-containing ceramic barium titanate. Tech. Phys. 42, 1097-1098 (1997)

11. I.V. Lisnevskaya, I.A. Bobrova, E.A. Bikyashev, T.G. Lupeiko, Interfacial reactions and properties of $\mathrm{Y}_{3} \mathrm{Fe}_{5} \mathrm{O}_{12} / \mathrm{Ba}_{1-x} \mathrm{~Pb}_{x} \mathrm{TiO}_{3}$ composites. Inorg. Mater. 42, 1147-1151 (2006)

12. J. Brančin, L. Medveck, Influence of mechanical activation of precursors on the structure and properties of donor doped $\mathrm{Ba}_{0.95} \mathrm{~Pb}_{0.05} \mathrm{TiO}_{3}$ ceramics. J. Mater. Sci. Lett. 21, 55-59 (2002)

13. A.I. Lebedev, I.A. Sluchinskaya, A. Erko, A.A. Veligzhanin, A.A. Chernyshov, XAFS studies of the local environment of $\mathrm{Pb}$ impurity atoms in barium, strontium, and calcium titanates. Phys. Solid State 51, 991-997 (2009) 
14. Landolt-Börnstein, Numerical Data and Functional Relationship in Science and Technology, Vol. III/28a (Springer, Berlin - Heidelberg, 1990), p. 228

15. J.H. Kim, S.H. Yoon, Y.H. Han, Effects of $\mathrm{Y}_{2} \mathrm{O}_{3}$ addition on electrical conductivity and dielectric properties of Ba-excess $\mathrm{BaTiO}_{3}$. J. Eur. Ceram. Soc. 27, 1113-1116 (2007)

16. Y. Park, H.G. Kim, The microstructure analysis of cerium - modified Barium Titanate having core-shell structured grains. Ceram. Int. 23, 329-336 (1997)

17. H. Takeda, H. Harinaka, T. Shiosaki, M.A. Zubair, C. Leach, R. Freer, T. Hoshina, T. Tsurumi, Fabrication and positive temperature coefficient of resistivity properties of semiconducting ceramics based on the $\mathrm{BaTiO}_{3}-\left(\mathrm{Bi}_{1} / 2 \mathrm{~K}_{1 / 2}\right) \mathrm{TiO}_{3}$ system. J. Eur. Ceram. Soc. 30, 555559 (2010)

18. R.A. Serway, W. Berlinger, K.A. Müller, R.W. Collins, Electron paramagnetic resonance of three manganese centers in reduced $\mathrm{SrTi}_{3}$. Phys. Rev. B 16, 4761-4768 (1977)

19. W. Li, J. Qi, Y. Wang, L. Li, Z. Gui, Doping behaviors of $\mathrm{Nb}_{2} \mathrm{O}_{5}$ and $\mathrm{Co}_{2} \mathrm{O}_{3}$ in temperature stable $\mathrm{BaTiO}_{3}$-based ceramics. Mat. Lett. 57, $1-5(2002)$

20. Q. Li, J. Qi, Y. Wang, Z. Gui, L. Li, Improvement of temperaturestable $\mathrm{BaTiO}_{3}$-based dielectrics by addition of $\mathrm{Li}_{2} \mathrm{CO}_{3}$ and $\mathrm{Co}_{2} \mathrm{O}_{3}$. J. Eur. Ceram. Soc. 21, 2217-2220 (2001)

21. S. Zhang, J.B. Lim, H.J. Lee, T.R. Shrout, Characterization of hard piezoelectric lead-free ceramics. IEEE Trans. Ultrason. Ferroelectr. Freq. Control 56, 1523-1527 (2009)

22. L.E. Cross, Relaxor ferroelectrics: an over view. Ferroelectrics 151, 305-320 (1994)

23. A.A. Bokov, Z.-G. Ye, Recent progress in relaxor ferroelectrics with perovskite structure. J. Mater. Sci. 41, 31-52 (2006)

24. G.A. Samara, The relaxational properties of compositionally disordered $\mathrm{ABO}_{3}$ perovskites. J. Phys. Condens. Matter 15, R367-R411 (2003)

25. M.E. Lines, A.M. Glass, Principle and application of ferroelectric and related materials (Clarendon, Oxford, 1977)

26. G. Arlt, H. Neumann, Internal bias in ferroelectric ceramics: origin and time dependence. Ferroelectrics 87, 109-120 (1988)

27. R. Bujakiewicz-Korońska, A. Kalvane, Y. Zhydachevskii, B. GarbarzGlos, W. Śmiga, L. Vasylechko, J. Czerwiec, A. Suchocki, A. Kamińska, W. Piekarczyk, Physical Properties of Ba0.95Pb0.05TiO3+0.1\%Co2O3. Ferroelectrics 436, 62-71 (2012)

28. M.C. Baehtz, H. Ehrenberg, H. Fuess, The synchrotron powder diffractometer at beamline B2 at HASYLAB/DESY: status and capabilities. J. Synchrotron Radiat. 11, 328-334 (2004)

29. L.G. Akselrud, P.Y. Zavalij, Y. Grin, V.K. Pecharsky, B. Baumgartner, E. Wölfel, Use of the CSD programme package for structure determination from powder data. Mater. Sci. Forum 133, 335-340 (1993)

30. G.W.H. Höhne, W.F. Hemminger, H.-J. Flammersheim, Differential Scanning Calorimetry (Springer, Berlin Heidelberg, 2003), p. 140

31. R. Pirc, R. Blinc, Off-center Ti model of barium titanate. Phys. Rev. B 70, 134107-1-8 (2004)

32. A. Bussmann-Holder, H. Beige, G. Völkel, Precursor effects, broken local symmetry, and coexistence of order-disorder and displacive dynamics in perovskite ferroelectrics. Phys. Rev. B 79, 184111-1-6 (2009)
33. A. Ziębińska, D. Rytz, K. Szot, M. Górny, K. Roleder, Birefringence above Tc in single crystals of barium titanate. J. Phys. Condens. Matter 20, 142202-1-5 (2008)

34. M. Takagi, T. Ishidate, Anomalous birefringence of cubic $\mathrm{BaTiO}_{3}$. Solid State Commun. 113, 423-426 (2000)

35. B. Zalar, V.V. Laguta, R. Blinc, NMR evidence for the coexistence of order-disorder and displacive components in Barium Titanate. Phys. Rev. Lett. 90, 037601-1-4 (2003)

36. B. Zalar, A. Lebar, J. Seliger, R. Blinc, NMR study of disorder in $\mathrm{BaTiO}_{3}$ and $\mathrm{SrTiO}_{3}$. Phys. Rev. B 71, 064107-1-12 (2005)

37. K. Wieczorek, A. Ziębińska, Z. Ujma, K. Szot, M. Górny, I. Franke, J. Koperski, S. Soszyński, K. Roleder, Electrostictive and piezoelectric effect in $\mathrm{BaTiO}_{3}$ and $\mathrm{PbZrO}_{3}$. Ferroelectrics 336, 6167 (2006)

38. J.H. Ko, S. Kojima, T.Y. Koo, J.H. Jung, C.J. Won, N.J. Hur, Elastic softening and central peaks in $\mathrm{BaTiO}_{3}$ single crystals above the cubic-tetragonal phase-transition temperature. Appl. Phys. Lett. 93, 102905-1-3 (2008)

39. J.H. Ko, T.H. Kim, K. Roleder, D. Rytz, S. Kojima, Precursor dynamics in the ferroelectric phase transition of barium titanate single crystals studied by Brillouin light scattering. Phys. Rev. B 84, 094123-1-9 (2011)

40. R. Migoni, H. Bilz, D. Bäuerle, Origin of raman scattering and ferroelectricity in oxid perovskites. Phys. Rev. Lett. 37, 1155-1158 (1976)

41. I. Ponomareva, L. Bellaiche, T. Ostapchuk, J. Hlinka, J. Petzelt, Terahertz dielectric response of cubic $\mathrm{BaTiO}_{3}$. Phys. Rev. B 77, 012102-1-4 (2008)

42. H. Bilz, G. Benedek, A. Bussmann-Holder, Theory of ferroelectricity: the polarizability model. Phys. Rev. B 35, 4840-4849 (1987)

43. J. Suchanicz, G. Stopa, K. Konieczny, D. Wcislo, M. Dziubaniuk, J. Rymarczyk, Uniaxial pressure effect on the dielectric properties of the $\mathrm{BaTiO}_{3}$ single crystals. Ferroelectrics 366, 3-10 (2008)

44. H.D. Megaw, Crystal structure of double oxides of the perovskite type. Proc. Phys. Soc. 58, 133-152 (1946)

45. Y. Yoshimura, M. Morioka, A. Kojima, N. Tokunaga, T. Koganezawa, $\mathrm{K}$. Tozaki, Reinterpretation of the unit cell evolution of $\mathrm{BaT}_{\mathrm{i}} \mathrm{O}_{3}$. Phys. Lett. A 367, 394-401 (2007)

46. C. Pawlaczyk, Domain wall dielectric response in the phase transition region in some ferroelectric. Ferroelectrics 140, 127$132(1993)$

47. K. Carl, K.H. Hardtl, Electrical after-effects in $\mathrm{Pb}(\mathrm{Ti}, \mathrm{Zr}) \mathrm{O}_{3}$ ceramics. Ferroelectrics 17, 473-486 (1978)

48. K.H. Hardtl, Electrical and mechanical losses in ferroelectric ceramics. Ceram. Int. 8, 121-127 (1982)

49. A.A. Bokov, Z.-G. Ye, Low-frequency dielectric spectroscopy of the relaxor ferroelectric $\mathrm{Pb}\left(\mathrm{Mg}_{1 / 3} \mathrm{Nb}_{2 / 3}\right) \mathrm{O}_{3}-\mathrm{PbTiO}_{3}$. Phys. Rev. B 65, 144112-1-10 (2002)

50. A.A. Bokov, Z.-G. Ye, Dielectric relaxation in relaxor ferroelectrics. J. Adv. Diel. 2, 1241010-1-24 (2012)

51. C.H. Park, Microscopic study on migration of oxygen vacancy in ferroelectric perovskite oxide. J. Phys. Soc. 42, S1420-S1424 (2003)

52. P. Erhart, R.-A. Eichel, P. Träskelin, K. Albe, Association of oxygen vacancies with impurity metal ions in lead titanate. Phys. Rev. B 76, 174116-1-12 (2007) 